\title{
Review
}

\section{Management of dementia: review of evidence}

\author{
Ratnatunge $S \mathbf{S}^{1}$, de Silva $V \mathbf{A}^{2}$ \\ Journal of the Ceylon College of Physicians, 2015, 46, 24-28
}

\section{Introduction}

Dementia is a progressive neurodegenerative disorder affecting approximately $1-2 \%$ of the population over the age of 65 years. ${ }^{1}$ There is deterioration in cognitive functions which affects several cognitive domains. The main cognitive domains affected are complex attention, executive functions, learning and memory, language, perceptual-motor and social cognition. ${ }^{1}$ The presentation depends on the subtype of dementia. The commonest type, Alzheimer's Disease typically presents with gradual onset progressive deterioration of memory and learning. The diagnosis of vascular dementia requires the confirmation of cerebrovascular disease in addition to establishing the diagnosis of dementia.

As the disease advances changes in mood and behaviour, psychotic phenomena and changes in personality become evident. These can be distressing and challenging both to the patient and the caregiver. These symptoms are commonly referred to by clinicians as behavioural and psychological symptoms of dementia or BPSDs.

Neuropathologically there are distinct types of dementia, although clinically it may be difficult to differentiate between them. The pathological changes of Alzheimer's disease consist of amyloid plaques, neurofibrillary tangles and neuronal loss. In early disease there is little or no macroscopic change but as the disease advances there is diffuse atrophy, flattening of sulci and enlargement of ventricles. In vascular dementia there are multiple infarcts caused by disease of small and medium sized cerebral vessels. In Lewy body dementia, a synucleinopathy, there are Lewy bodies in the brain stem and cortex. Mixed dementias where Alzheimer's disease coexist with vascular dementia or Lewy body disease are not uncommon.

\footnotetext{
${ }^{1}$ University Psychiatry Unit, National Hospital of Sri Lanka.

${ }^{2}$ Department of Psychiatry, Faculty of Medicine, University of Colombo, Sri Lanka.

Corresponding author: SSR,

E-mail: suhashini_sri@yahoo.com
}

Diagnosis is mainly based on clinical features. Neuroimaging is helpful in detecting other cerebral pathologies and identifying subtypes. Neuroimaging is not helpful in the diagnosis of early dementia. Neurocognitive tests are the most objective way of evaluating cognitive functions and serial tests are useful in monitoring the progression of the disease.

The objective of this review is to look at the evidence on the efficacy of pharmacological treatment and specific non-pharmacological interventions used for treatment of dementia.

\section{Methods}

We searched for articles using the key words dementia, cholinesterase inhibitors, memantine, non phamacological management and management. We searched the data bases of ALOIS (a comprehensive, open-access register of dementia studies), Cochrane database DARE (Database of Abstracts of Review of Effects) and PubMed. We looked at review articles and randomised controlled trials.

\section{Results}

\section{Pharmacotherapy}

The pharmacological management of dementia can be broadly conceptualized as managing the cognitive impairment with cognitive enhancers and the management of BPSDs.

\section{Cholinesterase inhibitors}

The place of cognitive enhancers have been widely studied in relation to Alzheimer's disease. The cholinesterase inhibitors (donepezil, rivastigmine and galantamine) now play a pivotal role in clinical practice and have also been successful in confronting the therapeutic nihilism attached to dementia. They work by increasing the survival of acetylcholine in the synapse and some even enhance the activity of the nicotinic-cholinergic receptor in the brain. Previously there was concern that these drugs do not alter disease process and the magnitude of their effect as disappointing. However recent RCTs indicate that cognitive functions do actually improve in one third and that more than half of patients are non-decliners. 


\section{Alzheimer's disease}

The three choline esterase inhibitors - donepezil, rivastigmine and galantamine have proven efficacy in mild to moderate Alzheimer's disease; even though there are minor differences in their pharmaco-dynamic profiles there is no difference in efficacy between the drugs. $^{2}$ Evidence from one large trial also showed donepezil to have less adverse effects than rivastigmine. ${ }^{3}$

\section{Vascular dementia}

Two large scale randomized trials provide evidence of benefit of donepezil in improving cognitive function, clinical global impression and activities of daily living in patients with mild to moderate vascular dementia after six months of treatment. ${ }^{4}$ Three large studies showed a statistically significant improvement in cognitive function in patients treated with rivastigmine for vascular dementia compared to placebo but there was no global improvement of change and no improvement in non-cognitive measures in the rivastigmine group. ${ }^{5}$

\section{Parkinson's disease dementia and Lewy body dementia}

Dementia occurs in about $40 \%$ of patients with Parkinson's disease especially in late onset cases with severe bradykinesia. A Cochrane Review of six randomised, double-blind, placebo-controlled trials assessing the efficacy of treatment with cholinesterase inhibitors showed significant improvement in global assessment, cognitive function, behavioural disturbance and activities of daily living rating scales in Parkinson's disease dementia. ${ }^{6}$

In a single trial there was no statistically significant improvement in patients with dementia with Lewy bodies (DLB) and further trials are needed to evaluate the place of choline esterase inhibitors in DLB.,7 Psychiatric disturbances which occur in Lewy body dementia are better treated with cholinesterase inhibitors than antipsychotics. However the evidence appears to be weak. ${ }^{8}$ Robust trials are needed in determining the efficacy of rivastigmine and other cholinesterase inhibitors in this disorder.

\section{Other dementias}

Fronto-temporal dementia, cerebral autosomal dominant arteriopathy with subcortical infarcts and leukoencephalopathy (CADASIL), dementia in multiple sclerosis, progressive supranuclear palsy and Huntington's disease are rare dementias accounting for less than $5 \%$ of all dementias. Current evidence shows that the efficacy of cholinesterase inhibitors on cognitive function and activities of daily living are unclear in these patient groups. ${ }^{9}$

\section{Memantine}

Memantine, an NMDA-glutamate receptor antagonist confers a neuro protective effect by antagonizing the excito-toxicity of neurons. It has been approved for treatment of moderate to severe Alzheimer's dementia (MMSE score <20).

\section{Alzheimer's dementia}

Memantine is effective in both mild-moderate and moderate-severe Alzheimer's dementia. ${ }^{10} \mathrm{~A}$ metaanalysis of nine studies found that memantine improved cognitive functions, activities of daily living and behavioural disturbance in patients with Alzheimer's dementia. ${ }^{11}$ However the effect size is small. Memantine is well tolerated by the elderly. The metaanalysis found that the rate of discontinuation and adverse events were similar to placebo.

There is some evidence that memantine is effective in the treatment of vascular dementia, Lewy body dementia and fronto-temporal dementias. Two six month studies have shown improvement in cognitive function and reduction of agitation in vascular dementia. ${ }^{10}$ Two RCTs have shown small improvement in cognitive functions in vascular dementia. ${ }^{10}$ Two RCTs showed memantine to be marginally superior to placebo in the treatment of fronto-temporal dementia as assed by clinical global impression scores. ${ }^{12}$

\section{Combination treatment}

Because memantine and cholinesterase inhibitors have different mechanisms of action combination treatment has been investigated in several RCTs. Meta-analysis of four RCTs which investigated the efficacy and safety of memantine $20 \mathrm{mg}$ a day and an cholinesterase inhibitor found a small improvement in cognitive functions and good tolerability. ${ }^{13}$

\section{Other medications}

Four studies including two large randomized controlled trials did not indicate statins to be beneficial in the treatment of cognitive decline in Alzheimer's disease. ${ }^{14}$ Aspirin is widely prescribed to patients with vascular dementia. However to date there is no good evidence to state that aspirin is beneficial in patients with vascular dementia. ${ }^{15}$

\section{Pharmacotherapy for BPSD}

\section{Antidepressants}

Evidence was reviewed for the effectiveness and safety of antidepressants in the treatment of agitation and psychosis for patients with dementia. Two studies found that selective serotonin reuptake inhibitors (SSRI) sertraline and citalopram can reduce agitation and 
psychosis. SSRIs and trazadone are better tolerated than antipsychotics. ${ }^{16} \mathrm{~A}$ recent review of eight trials of SSRIs and three of trazadone showed benefit in treating BPSDs. Of the 19 trials reviewing tolerability 14 showed a favorable outcome with regards to side effects. ${ }^{17}$

\section{Atypical antipsychotics}

Atypical antipsychotics have been the therapeutic standard in the management of BPSDs. Evidence suggest that both risperidone and olanzapine are effective in aggression and risperidone claiming an additional benefit of reducing psychosis. Modest efficacy is evident, however recent safety concerns regarding serious cerebrovascular events and mortality highlights that neither drug should be routinely prescribed and given only in selective cases where the aggression and psychosis causes severe distress to the patient and family and there is overt risk to both parties. $^{18}$

\section{Anticonvulsants}

Evidence supports the use of carbamazepine in agitation and aggression in dementia although the evidence base is small and problematic side effects limits its use.$^{18}$ On the basis of current evidence it can be concluded that valproate is not recommended for agitation and aggression in dementia and the adverse effects of valproate appear to be poorly tolerated. ${ }^{19}$ There have been studies of several new antiepiletics (levetiracetam, oxcarbazepine, gabapentin, topiramate and lamotrigine) in the management of BPSDs. Considering the lack of high-quality data, these medications are not recommended. ${ }^{20}$

\section{Non-pharmacological management of BPSD}

Several non-pharmacological interventions have been investigated for the management of dementia. Interventions with evidence of efficacy in the management of agitation include group activities involving persons with dementia, music therapy and sensory interventions. Five RCTs conducted in care homes reported that group activities reduce agitation. Three RCTs which investigated music therapy which involves listening to music and singing found it to be effective. Five RCTs investigated sensory interventions which address under stimulation in people with dementia and found them to be effective.

\section{Discussion}

This review appraises the current evidence for the pharmacological management of dementia. Current evidence support the efficacy of cholinesterase inhibitors and memantine in the treatment of different subtypes of dementia.
Current evidence suggests that deterioration in cognition, behavior and functioning should no longer be considered part of the natural history of aging as new therapeutic options have been shown to make significant inroads into stymying their impact and heralding a paradigm shift in our approach to the "management of aging".

With the world population aging prevention and treatment of dementia is a priority. Identification of biomarkers, which aid early diagnosis, is still in its infancy. About $50 \%$ with mild cognitive impairment (MCl) go on to develop Alzheimer's dementia. However there is no consensus on whether $\mathrm{MCl}$ should be treated with cholinesterase inhibitors.

Interventions which reduce the risk of dementia are mainly healthy life style changes such as giving up smoking and excessive alcohol consumption, promoting physical activity, proper diet, and the management of conditions such as hypertension, diabetes and obesity. ${ }^{21}$ In addition cognitive stimulation delays or prevents onset of dementia.

Most treatment guidelines recommend starting cholinesterase inhibitors as first line treatment of Alzheimer's dementia. The growing pool of evidence supports its efficacy in vascular, Lewy body and Parkinson's disease dementia too. Unlike in stroke there is no evidence to say that statins or aspirin do have an effect in the incidence of vascular dementia or its progression. ${ }^{14}$ There is little evidence which supports the superiority of one cholinesterase inhibitor over others. Therefore choice depends on availability, cost, tolerability, frequency (once daily for donepezil and twice daily for the oral forms of rivastigmine and galantamine) and ease of administration (rivastigmine is available as a $4.6 \mathrm{mg} / 24$ hours patch and galantamine as a 8 mg extended release preparation).

Donepezil is commenced at a dose of $5 \mathrm{mg} /$ day but can be increased to $10 \mathrm{mg} /$ day in 4-6 weeks. ${ }^{22}$ Evidence shows that $10 \mathrm{mg}$ is more effective than $5 \mathrm{mg}$. Main side effects are due to peripheral inhibition of acetylcholinesterase which causes gastrointestinal effects such as nausea, vomiting and diarrhoea. Bradycardia or heart block can occur rarely. ECG before starting treatment is recommended.

Rivastigmine is commenced at $1.5 \mathrm{mg}$ twice a day and increased by $3 \mathrm{mg}$ every 2 weeks. ${ }^{22}$ Maximum recommended dose is $6 \mathrm{mg}$ twice a day. Slow titration is required because of the risk of side effects. Side effects are the same as in donepezil. Rivastigmine is available as a transdermal patch which is applied once a day. Dose increase should be done after a minimum of 4 weeks. 
Galantamine is started at $4 \mathrm{mg}$ twice daily and increased every 4 weeks to a maximum of $12 \mathrm{mg}$ twice a day. ${ }^{22}$

Memantine is commenced at $5 \mathrm{mg}$ a day and increased by $5 \mathrm{mg}$ every week. ${ }^{22}$ Doses over $5 \mathrm{mg}$ should be given twice a day. Maximum dose is $10 \mathrm{mg}$ twice a day. This is well tolerated and cardiac side effects are minimal.

Although clinical trials show that cholinesterase inhibitors and memantine are effective in improving cognitive functions there is no consensus on if these translate to clinically meaningful changes. Majority of studies had used the ADAS-cog as their assessment tool. The average annual rate of decline in untreated patients is between 6-12 points on the ADAS-cog. A four point improvement in the ADAS-cog is considered clinically meaningful. ${ }^{23}$

Most RCTs show that the cognitive functions improve by a few points. However some studies have reported improvement in activities of daily living, mood, agitation and other behavioural and psychological symptoms of dementia.

Discontinuing cholinesterase inhibitors in patients with moderate to severe $A D$ may lead to worsening of cognitive function and greater functional impairment. Therefore unless cost or side effects are problematic, treatment should be continued. Patients benefit from serial assessments of cognitive functions and level of functioning. This enables the clinician to identify those who respond poorly to cholinesterase inhibitors. In such patients changing to memantine or combination of cholinesterase inhibitors with memantine should be considered.

Over $60 \%$ of patients with dementia develop BPSD. They present as agitation, aggression, apathy, depression, psychotic symptoms, sexual disinhibition etc. BPSD are the most significant determinant in generating carer stress and burden and a likelihood of institutionalization. There is a weak correlation between the level of cognitive impairment and the occurrence and severity of BPSDs. However there is a strong association between the presence of these symptoms and the degree of functional impairment and ADL. Unlike cognitive symptoms which are progressive BPSD run a fluctuating course. It is well described that apart from the brain pathology (intrinsic factors) environmental setting, level of stimulation, activity triggers (extrinsic factors) and care giver factors contribute toward the development of these symptoms. Therefore for the effective management of BPSD an amalgamation of both pharmacological and non pharmacological approaches are crucial.

Parkinson's disease dementia and Lewy body dementia are particularly difficult to manage. In Lewy body dementia visual hallucinations and psychotic symptoms are common and anti-Parkinson's medication can worsen BPSD. Despite little evidence cholinesterase inhibitors are recommended. Antipsychotics can worsen autonomic symptoms and are therefore best avoided.

Although we have mainly reviewed the pharmacotherapy of dementia the management involves much more. The ultimate goal is to ensure a good quality of life to the patient and to carers. This involves a multidisciplinary input from the health and social care services and the family.

\section{References}

1. American Psychiatric Association. Diagnostic and statistical manual of mental disorders: DSM-5. Washington, D.C: American Psychiatric Association 2013.

2. Tan CC, Yu JT, Wang HF, Tan MS, Meng XF, Wang C, et al. Efficacy and safety of donepezil, galantamine, rivastigmine, and memantine for the treatment of Alzheimer's disease: a systematic review and meta-analysis. Journal of Alzheimer's Disease 2014; 41(2): 615-31.

3. Birks J. Cholinesterase inhibitors for Alzheimer's disease. Cochrane Database of Systematic Reviews. 2006 (1): CD005593.

4. Malouf R, Birks J. Donepezil for vascular cognitive impairment. Cochrane Database of Systematic Reviews. 2004 (1): CD004395.

5. Birks J, McGuinness B, Craig D. Rivastigmine for vascular cognitive impairment. Cochrane Database of Systematic Reviews 2013; 5: CD004744.

6. Rolinski M, Fox C, Maidment I, McShane R. Cholinesterase inhibitors for dementia with Lewy bodies, Parkinson's disease dementia and cognitive impairment in Parkinson's disease. Cochrane Database of Systematic Reviews 2012; 3: CD006504.

7. Stinton C, McKeith I, Taylor JP, Lafortune L, Mioshi E, MakE, et al. Pharmacological Management of Lewy Body Dementia: A Systematic Review and Meta-Analysis. American Journal of Psychiatry 2015 Aug 1; 172(8): 731-42.

8. Wild R, Pettit T, Burns A. Cholinesterase inhibitors for dementia with Lewy bodies. Cochrane Database of Systematic Reviews 2003 (3): CD003672.

9. Li Y, Hai S, Zhou Y, Dong BR. Cholinesterase inhibitors for rarer dementias associated with neurological conditions. Cochrane Database of Systematic Reviews 2015; 3: CD009444.

10. McShane R, Areosa Sastre A, Minakaran N. Memantine for dementia. Cochrane Database of Systematic Reviews 2006 (2): CD003154.

11. Matsunaga S, Kishi T, Iwata N. Memantine monotherapy for Alzheimer's disease: a systematic review and metaanalysis. PloS One 2015; 10(4): e0123289.

12. Kishi T, Matsunaga S, Iwata N. Memantine for the treatment of frontotemporal dementia: a meta-analysis. Neuropsychiatric Disease and Treatment 2015; 11: 2883-5. 
13. Farrimond LE, Roberts E, McShane R. Memantine and cholinesterase inhibitor combination therapy for Alzheimer's disease: a systematic review. BMJ Open 2012; 2(3).

14. McGuinness B, Craig D, Bullock R, Malouf R, Passmore P. Statins for the treatment of dementia. Cochrane Database of Systematic Reviews 2014; 7: CD007514.

15. Williams PS, Rands G, Orrel M, Spector A. Aspirin for vascular dementia. Cochrane Database of Systematic Reviews 2000 (4): CD001296.

16. Seitz DP, Adunuri N, Gill SS, Gruneir A, Herrmann N, Rochon P. Antidepressants for agitation and psychosis in dementia. Cochrane Database of Systematic Reviews 2011 (2): CD008191.

17. Henry G, Williamson D, Tampi RR. Efficacy and tolerability of antidepressants in the treatment of behavioral and psychological symptoms of dementia, a literature review of evidence. American Journal of Alzheimers Disease and other Dementias 2011 May; 26(3): 169-83.

18. Ballard C, Waite J. The effectiveness of atypical antipsychotics for the treatment of aggression and psychosis in Alzheimer's disease. Cochrane Database of Systematic Reviews 2006 (1): CD003476.

19. Lonergan $\mathrm{E}$, Luxenberg J. Valproate preparations for agitation in dementia. Cochrane Database of Systematic Reviews 2009 (3): CD003945.

20. Gallagher D, Herrmann N. Antiepileptic drugs for the treatment of agitation and aggression in dementia: do they have a place in therapy? Drugs 2014 Oct; 74(15): 1747-55.

21. National Institute for Health and Clinical Excellence. Dementia: Supporting people with dementia and their carers in health and social care. National Institute for Health and Clinical Excellence. London 2006.

22. Stevem M.Stahl. Stahl's essential psychopharmacology: prescriber's guide. $5^{\text {th }}$ Ed. Cambridge University Press 2015

23. Stern RG, Mohs RC, Davidson M, Schmeidler J, Silverman J, Kramer-Ginsberg E, et al. A longitudinal study of Alzheimer's disease: measurement, rate, and predictors of cognitive deterioration. American Journal of Psychiatry 1994 Mar; 151(3): 390-6. 